\title{
Comparison results for solutions of elliptic problems via symmetrization
}

by

\author{
Angelo ALVINO, Guido TROMBETTI
}

Dipartimento di Matematica e Applicazioni $R$. Caccioppoli.

Pierre-Louis LIONS

Ceremade, Université Paris-Dauphine.

and

\section{Silvano MATARASSO}

Dipartimento di Matematica- CIRAM, Università di Bologna.

ABSTRACT. - We give some comparison results for solutions of Dirichlet problems of elliptic equations and variational inequalities by means of Schwarz symmetrization.

(C) 1999 L'Association Publications de l'Institut Henri Poincaré. Published by Elsevier B.V. All rights reserved

Key words: Schwarz symmetrization, elliptic PDE, variational inequalities.

RÉSUMÉ. - Nous donnons divers résultats de comparaison obtenus par la symétrisation de Schwarz pour les solutions d'équations et inéquations elliptiques.

(C) 1999 L'Association Publications de l'Institut Henri Poincaré. Published by Elsevier B.V. All rights reserved

\section{INTRODUCTION}

If $\Omega$ is an open bounded subset of $R^{N}$ and $\varphi \in L^{1}(\Omega)$, the distribution function of $\varphi$ is the function $\mu_{\varphi}$ defined by

$$
\mu_{\varphi}(t)=|\{x \in \Omega:|\varphi(x)|>t\}|, t \in R^{+} .
$$

Classification A.M.S.: $35 \mathrm{~J} 25,35 \mathrm{~J} 85$.

$$
\begin{gathered}
\text { Annales de l'Institut Henri Poincaré - Analyse non linéaire - 0294-1449 } \\
\text { Vol. 16/99/02/ }
\end{gathered}
$$


For $s \in[0,|\Omega|]$ we set

$$
\varphi^{*}(s)=\sup \left\{t: \mu_{\varphi}(t) \geq s\right\}
$$

$\varphi^{*}$ is the decreasing rearrangement of $\varphi$.

Let $\Omega^{\sharp}$ denote the ball of $R^{N}$ centered at zero whose measure is $|\Omega|$, we define

$$
\varphi^{\sharp}(x)=\varphi^{*}\left(\omega_{N}|x|^{N}\right), \quad x \in \Omega^{\sharp},
$$

where $\omega_{N}$ is the volume of unit ball in $R^{n} ; \varphi^{\sharp}$ is known as the decreasing, spherically symmetric rearrangement of $\varphi$. Finally we denote by $\varphi_{*}(s)=\varphi^{*}(|\Omega|-s)$ and $\varphi_{\sharp}(x)=\varphi_{*}\left(\omega_{N}|x|^{N}\right)$ respectively the increasing rearrangement and the increasing spherically symmetric rearrangement of $\varphi$.

We consider the linear elliptic differential operator

$$
A u=-\sum_{i, j=1}^{N}\left(a_{i j}(x) u_{x_{i}}\right)_{x_{j}}+\sum_{i=1}^{N} b_{i}(x) u_{x_{i}}+\sum_{i=1}^{N}\left(d_{i}(x) u\right)_{x_{i}}+c(x) u
$$

where the coefficients are measurable and bounded. Moreover it is assumed that for almost all $x \in \Omega$

$$
\begin{gathered}
\sum_{i, j=1}^{N} a_{i j}(x) \xi_{i} \xi_{j} \geq|\xi|^{2}, \quad \forall \xi \in \mathbf{R}^{N} \\
\sum_{i=1}^{N}\left|b_{i}(x)\right|^{2} \leq B^{2} \\
\sum_{i=1}^{N}\left|d_{i}(x)\right|^{2} \leq D^{2} \\
c(x) \geq 0
\end{gathered}
$$

where $B, D$ are non negative constants. If $u \in H_{0}^{1}(\Omega)$ is a weak solution of the homogeneous Dirichlet problem

$$
A u=f \text { in } \Omega, u=0 \text { on } \partial \Omega,
$$

with $f \in L^{2}(\Omega)$, any $L^{p}$ or Orlicz norm of $u$ can be regarded as a functional of the data of problem (5); we fix some constraints on these data (see conditions (1)-(4)) and look for those for which a norm of $u$ achieves 
its maximum. In order to describe the kind of result we have in mind we recall what happens when the operator $A$ has the following simple structure

$$
-\sum_{i, j=1}^{N}\left(a_{i j}(x) u_{x_{i}}\right)_{x_{j}}+c u .
$$

In this case if $v \in H_{0}^{1}\left(\Omega^{\sharp}\right)$ is the weak solution of the problem

$$
-\Delta v+c_{\sharp}(x) v=f^{\sharp} \text { on } \Omega^{\sharp}, v=0 \text { on } \partial \Omega^{\sharp},
$$

we can estimate the concentration of $u$ by the concentration of $v$,

$$
\int_{0}^{s} u^{*} \leq \int_{0}^{s} v^{*}, \forall s \in[0,|\Omega|] .
$$

The result (7) may be improved when $c \equiv 0$; in this case we have

$$
u^{*}(s) \leq v^{*}(s), \forall s \in[0,|\Omega|] .
$$

The pointwise estimate (8) was first obtained by G. Talenti [14]; for some extensions of this result and for a proof of the integral estimate (7) see [2], [3], [4], [6], [8], [12], [15].

By means of (7) it is possible to estimate any $L^{p}$ norm of $u$ by the same norm of the solution $v$ of (6)

$$
\|u\|_{L^{p}} \leq\|v\|_{L^{p}}, \quad 1 \leq p \leq+\infty
$$

so we can assert that any $L^{p}$ norm of the solution $u$ of problem (5) achieves its maximum when the data of the problem (coefficients, domain and known term) are spherically symmetric.

This kind of problem becomes more involved when we take first order terms into account in the structure of the operator $A$.

Namely if $u \in H_{0}^{1}(\Omega)$ solves the following Dirichlet problem

$$
\left\{\begin{array}{l}
L u=-\sum_{i, j=1}^{N}\left(a_{i j}(x) u_{x_{i}}\right)_{x_{j}}+\sum_{i=1}^{N} b_{i}(x) u_{x_{i}}+c(x) u=f, \text { on } \Omega \\
u=0, \text { on } \partial \Omega
\end{array}\right.
$$

and $v \in H_{0}^{1}\left(\Omega^{\sharp}\right)$ is the weak solution of

$$
\left\{\begin{array}{l}
-\Delta v+B \sum_{i=1}^{N} \frac{x_{i}}{|x|} v_{x_{i}}+c_{\sharp}(x) v=f^{\sharp}, \text { on } \Omega^{\sharp}, \\
v=0, \text { on } \partial \Omega^{\sharp},
\end{array}\right.
$$

Vol. 16, $n^{\circ} 2-1999$. 
the following estimate holds for all $s \in[0,|\Omega|]$ (see [2], [5])

$$
\int_{0}^{s} \exp \left(-B \omega_{N}^{-1 / N} \sigma^{1 / N}\right) u^{*}(\sigma) \mathrm{d} \sigma \leq \int_{0}^{s} \exp \left(-B \omega_{N}^{-1 / N} \sigma^{1 / N}\right) v^{*}(\sigma) \mathrm{d} \sigma .
$$

Obviously, (12) implies that

$$
\|u\|_{L^{\infty}} \leq\|v\|_{L^{\infty}}
$$

however it is no use if we ask to estimate $L^{p}$ norms of $u$ by the same norm of $v$. In order to obtain (9) it is essential to prove the integral estimate (7) which does not hold as shown in [2].

The aim of this paper is to show that we can estimate the concentration of the solution $u$ of (10) by the concentration of the solution of a suitable spherically symmetric problem that is different from (11). More precisely let $\gamma$ be a spherically symmetric function, increasing with respect to $\rho=|x|$, such that

$$
\exp (-B|x|) c_{\sharp}(x) \geq \gamma(x) \geq 0 ;
$$

if $z \in H_{0}^{1}\left(\Omega^{\sharp}\right)$ is the weak solution of the problem

$$
\left\{\begin{array}{l}
S z=-\Delta z+B \sum_{i=1}^{N} \frac{x_{i}}{|x|} z_{x_{i}}+\exp (B|x|) \gamma(x) z=f^{\ddagger}, \text { in } \Omega^{\sharp}, \\
z=0, \text { on } \partial \Omega^{\sharp},
\end{array}\right.
$$

then we have (see Theorem 2.1)

$$
\int_{0}^{s} u^{*}(\sigma) \mathrm{d} \sigma \leq \int_{0}^{s} z^{*}(\sigma) \mathrm{d} \sigma, s \in[0,|\Omega|] .
$$

In other words, the desired comparison holds provided we choose the zero order term $\widetilde{c}(x)=\exp (B|x|) \gamma(x)$ in such a way that $\widetilde{c}(x)$ is spherically symmetric, $0 \leq \widetilde{c}(x) \leq c_{\sharp}(x)$ on $\Omega^{\sharp}, \widetilde{c}$ is increasing and $\exp (-B|x|) \widetilde{c}(x)$ is increasing. From this comparison result, via a duality argument, it is possible to infer a similar result for the following problem

$$
\left\{\begin{array}{l}
-\sum_{j=1}^{N}\left(\sum_{i=1}^{N} a_{i j}(x) u_{x_{i}}+b_{j}(x) u\right)_{x_{j}}+c u=f, \text { on } \Omega, \\
u=0, \text { on } \partial \Omega .
\end{array}\right.
$$

We also give a direct proof of this result in order to apply it to variational inequalities (see [7], [13], [5] for related results). 


\section{MAIN RESULTS: ELLIPTIC EQUATIONS}

Our first goal is the proof of the comparison result (7) for a solution of problem (10).

THEOREM 2.1. - Let $u \in H_{0}^{1}(\Omega)$ be the weak solution of (10) where the coefficients satisfy conditions (1), (2), (14) and $f \in L^{2}(\Omega)$; moreover let $z \in H_{0}^{1}\left(\Omega^{\sharp}\right)$ be the weak solution of problem (15). Then, (16) holds. Furthermore, if $\gamma(x)=0$ when $|x| \leq R_{0}$, then

$$
u^{\sharp}(x) \leq v^{\sharp}(x),|x| \leq R_{0} .
$$

REMARK 2.1. - If the function $\exp (-B|x|) c_{\sharp}(x)$ is increasing with respect to $|x|$ we can set $\gamma(x)=\exp (-B|x|) c_{\sharp}(x)$. In this case, the problem (15) is exactly the problem (11): so, by means of Theorem 2.1, we obtain the integral estimate (16) instead of the weaker estimate (12). On the other hand if the function $\exp (-B|x|) c_{\sharp}(x)$ does not satisfy this monotonicity property, we compare $u$ with the solution of (15) that is greater than the solution of (11); this implies that we could sharply estimate $u$ by $v$ rather than by $z$ (see $L^{\infty}$ estimate (13)). However since (16) holds, we can estimate any $L^{p}$ norm of $u$ by the same norm of $z$ too.

We begin by showing the following properties of the solution of (15):

LEMMA 2.1. - The solution $z \in H_{0}^{1}\left(\Omega^{\sharp}\right)$ of (15) is non negative, spherically symmetric and decreasing with respect to $|x|$.

We have to prove that $z$ is decreasing with respect to $|x|$, because the other properties can be easily derived from the maximum principle. For simplicity we assume that $f^{\sharp}$ and $\gamma$ are sufficiently smooth.

Setting $z(\rho)=z(x), f(\rho)=f^{\sharp}(x), \gamma(\rho)=\gamma(x)$, we have

$$
\left\{\begin{array}{l}
-z^{\prime \prime}+z^{\prime}\left(B-\frac{N-1}{\rho}\right)+\exp (B \rho) \gamma z=f, \rho \in[0, R] \\
z^{\prime}(0)=0, z(R)=0
\end{array}\right.
$$

where $R$ is the radius of $\Omega^{\sharp}$. Differentiating with respect to $\rho$ and letting $w=z^{\prime}$, we deduce

$$
\left\{\begin{array}{l}
-w^{\prime \prime}+\left(B-\frac{N-1}{\rho}\right) w^{\prime}+\left(\exp (B \rho) \gamma+\frac{N-1}{\rho^{2}}\right) w=f^{\prime}-[\exp (B \rho) \gamma]^{\prime} z \\
w^{\prime}(0)=0, w(R) \leq 0
\end{array}\right.
$$

Since $\exp (B \rho) \gamma$ is increasing, the right hand side in the equation (18) is non positive; then by maximum principle $w=z^{\prime} \leq 0$. 
When $f^{\sharp}$ and $\gamma$ are not smooth we can proceed by approximation; however a direct proof could be given replacing in the above argument derivatives by difference quotients.

Proof of Theorem 2.1. - We set

$$
\begin{gathered}
k_{N}=N \omega_{N}^{1 / N}, \quad e(s)=\exp \left(B \omega_{N}^{-1 / N} s^{1 / N}\right), \\
k(s)=\frac{1}{k_{N}^{2}} s^{2 / N-2} e(s) .
\end{gathered}
$$

Let $u(s)=u^{*}(s)$ denote the rearrangement of the solution $u(x) \in H_{0}^{1}(\Omega)$ of (10); then we have (see [5] and the Appendix)

$$
-u^{\prime}(s) \leq k(s) \int_{0}^{s} e^{-1}\left(f^{*}-c_{*} u\right)
$$

and then, by (14),

$$
-u^{\prime}(s) \leq k(s) \int_{0}^{s}\left(e^{-1} f^{*}-\gamma_{*} u\right) .
$$

Taking into account Lemma 2.1, a direct computation yields:

$$
-z^{\prime}(s)=k(s) \int_{0}^{s}\left(e^{-1} f^{*}-\gamma_{*} z\right),
$$

where $z(s)$ denotes the rearrangement $z^{*}$ of the solution $z \in H_{0}^{1}\left(\Omega^{\sharp}\right)$ of (15).

Setting $w=u-z$, we obtain, from (20) and (21),

$$
\left\{\begin{array}{l}
-w^{\prime}(s)+k(s) \int_{0}^{s} \gamma_{*} w \leq 0 \\
w(0)=0, w^{\prime}(|\Omega|)=0
\end{array}\right.
$$

We claim that (22) implies that

$$
\left.W(s)=\int_{0}^{s} \gamma_{*} w \leq 0, s \in\right] 0,|\Omega|[.
$$

Indeed, let us assume that (23) does not hold; since $w^{\prime}(|\Omega|)=0$, there exists an interval $[a, b] \subseteq[0,|\Omega|]$ such that

i) $W(s)>0, s \in] a, b[$,

ii) $W(a)=W^{\prime}(b)=0$. 
In particular, we can deduce from (22) and i) that we have, for $s \in] a, b[$ :

$$
-w^{\prime}(s) W(s)+k(s) W^{2}(s)<0 ;
$$

integrating on $[a, b]$ and taking into account the boundary conditions satisfied by $W$ (see ii)), since $\gamma_{*}(b)>0$, we get

$$
0 \geq-\int_{a}^{b} w^{\prime} W+\int_{a}^{b} k W^{2}=\int_{a}^{b} \gamma_{*} w^{2}+\int_{a}^{b} k W^{2}
$$

which contradicts i); hence (23) holds.

If $\gamma_{*} \equiv 0$ on $\left[0, s_{0}\right],(23)$ becomes

$$
\int_{s_{0}}^{s} \gamma_{*} u \leq \int_{s_{0}}^{s} \gamma_{*} z, s \in\left[s_{0},|\Omega|\right],
$$

from which we deduce as is well known,

$$
\int_{s_{0}}^{s} u \leq \int_{s_{0}}^{s} z, s \in\left[s_{0},|\Omega|\right] .
$$

This completes the proof of theorem 2.1, in the case $s_{0}=0$.

If $s_{0}>0$, (24) yields

$$
u\left(s_{0}\right) \leq z\left(s_{0}\right)
$$

on the other hand, (22) implies

$$
-u^{\prime}(s) \leq-z^{\prime}(s), s \in\left[0, s_{0}\right]
$$

and we obtain the pointwise estimate (17) which completes the proof.

Now, we consider the following Dirichlet problem

$$
\left\{\begin{array}{l}
L^{*} u=-\sum_{i=1}^{N}\left(\sum_{j=1}^{N} a_{i j}(x) u_{x_{j}}+b_{i}(x) u\right)_{x_{i}}+c(x) u=f \\
u=0, \text { on } \partial \Omega
\end{array}\right.
$$

and the the symmetrized one

$$
\left\{\begin{array}{l}
S^{*} v=-\Delta v-B \sum_{i=1}^{N}\left(\frac{x_{i}}{|x|} v\right)_{x_{i}}+\exp (B|x|) \gamma(x) v=f^{\sharp}, \\
v=0, \text { on } \partial \Omega^{\sharp} .
\end{array}\right.
$$

Vol. $16, n^{\circ} 2-1999$. 
We have that $v$ is non negative, spherically symmetric and decreasing with respect to $\rho=|x|$. Indeed the function

$$
V(x)=\exp (B|x|) v(x)
$$

satisfies the following equation

$$
-\left(\exp (-B|x|) V_{x_{i}}\right)_{x_{i}}+\gamma(x) V=f^{\sharp}
$$

If we set $V(\rho)=V(x), \gamma(\rho)=\gamma(x), f(\rho)=f^{\ddagger}(x)$, where $\rho=|x|$, we find

$$
\left[-V^{\prime \prime}+V^{\prime}\left(B-\frac{N-1}{\rho}\right)\right] \exp (-B \rho)+\gamma(\rho) V(\rho)=f
$$

differentiating with respect to $\rho$ and setting $Z=V^{\prime} \exp (-B \rho)$, we obtain

$$
\left\{\begin{array}{l}
-Z^{\prime \prime}-\frac{N-1}{\rho} Z^{\prime}+Z\left[\gamma(\rho) \exp (B \rho)+\frac{N-1}{\rho^{2}}\right]=f^{\prime}-\gamma^{\prime} V \\
Z(0)=0, Z(R) \leq 0
\end{array}\right.
$$

where $R$ is the radius of $\Omega^{\sharp}$. By the maximum principle we deduce that $Z \leq 0$ i.e. $V^{\prime} \leq 0$; so $V$ and $v$ are decreasing with respect to $\rho$.

REMARK 2.2. - We have

$$
\begin{aligned}
v(x)= & \frac{1}{N \omega_{N}} \exp (-B|x|) \int_{|x|}^{\left(|\Omega| / \omega_{N}\right)^{1 / N}} t^{1-N} \\
& \exp (B t) \int_{0}^{\omega_{N} t^{N}}\left(f^{*}(s)-\gamma_{*}(s) e(s) v^{*}(s)\right) d s
\end{aligned}
$$

and thus

$$
V^{\prime}=(v \exp (B \rho))^{\prime}=-\frac{\rho^{1-N}}{N \omega_{N}} \exp (B \rho) \int_{0}^{\omega_{N} \rho^{N}}\left(f^{*}(s)-\gamma_{*}(s) e(s) v^{*}(s)\right) d s \leq 0
$$

so we have the following inequality

$$
\int_{0}^{\omega_{N} \rho^{N}}\left(f^{*}(s)-\gamma_{*}(s) e(s) v^{*}(s)\right) d s \geq 0
$$

We now recall some properties of rearrangements we shall use in the sequel: 
- if $f$ and $g$ are measurable functions defined on a set $\Omega$ then (Hardy inequality (see [10])):

$$
\int_{0}^{|\Omega|} f^{*} g_{*} \leq \int_{\Omega} f g \leq \int_{0}^{|\Omega|} f^{*} g^{*}
$$

- the following are equivalent (see [1] theorem 2.1)
a) $\int_{0}^{s} f^{*} \leq \int_{0}^{s} g^{*}, \forall s \in[0,|\Omega|]$,
b) $\int_{\Omega} f \varphi \leq \int_{0}^{|\Omega|} g^{*} \varphi^{*}, \forall \varphi \in L_{+}^{\infty}(\Omega)$,
c) $\int_{\Omega} f^{*} \varphi^{*} \leq \int_{0}^{|\Omega|} g^{*} \varphi^{*}, \forall \varphi \in L_{+}^{\infty}(\Omega)$,
d) $\int_{\Omega} F(f) \leq \int_{\Omega} F(g)$

for all convex, non negative, Lipschitz function $F$ such that $F(0)=0$.

Theorem 2.2. - Suppose that (1), (2), (14) are satisfied, then we have the inequality

$$
\int_{0}^{s} u^{*} \leq \int_{0}^{s} v^{*}, s \in[0,|\Omega|],
$$

where $u$ and $v$ are solutions, respectively, of (25) and (26).

We use a duality argument. If $g \in L^{2}(\Omega)$, let $z \in H_{0}^{1}(\Omega)$ be the solution of the equation $L z=g$. Moreover, let $w \in H_{0}^{1}\left(\Omega^{\sharp}\right)$ be the solution of the symmetrized equation $S w=g^{\sharp}$. We have

$$
\begin{gathered}
\int_{\Omega} g u=\int_{\Omega} u L z=\int_{\Omega} z L^{*} u=\int_{\Omega} z f \\
\leq \int_{\Omega^{\sharp}} f^{\sharp} z^{\sharp} \text { (by Hardy inequality) } \\
\leq \int_{\Omega^{\sharp}} f^{\sharp} w \text { (by theorem } 2.1 \text { and property c)) } \\
=\int_{\Omega^{\sharp}} w S^{*} v=\int_{\Omega^{\sharp}} v S w=\int_{\Omega^{\sharp}} v g^{\sharp} .
\end{gathered}
$$

Then we have, since $v=v^{\sharp}$,

$$
\int_{\Omega} u g \leq \int_{\Omega^{\sharp}} v^{\sharp} g^{\sharp}, \forall g \in I^{2}(\Omega) ;
$$

by property b) we get the result. 


\section{MAIN RESULTS: VARIATIONAL INEQUALITIES}

In this section we extend the results of section 2 to the case of variational inequalities. The analogue of theorem 2.1 is proven in a very similar way to the proof made in the case of equations. On the other hand, in order to extend theorem 2.2 , we need a somewhat different proof since we cannot proceed by a duality argument.

Let $f \in L^{2}(\Omega)$, we consider the following problem

$$
\left\{\begin{array}{l}
\prec L u, \varphi-u \succ \geq \int_{\Omega} f(\varphi-u), \forall \varphi \in H_{0}^{1}(\Omega), \varphi \geq 0 \\
u \geq 0 ;
\end{array}\right.
$$

it is known (see [11] for example) that the variational inequality (29) has a unique solution $u \in H_{0}^{1}(\Omega)$.

In addition let $z \in H_{0}^{1}\left(\Omega^{\sharp}\right)$ be the solution of the following symmetrized problem

$$
\left\{\begin{array}{l}
\prec S z, \varphi-z \succ \geq \int_{\Omega^{\sharp}} f^{\sharp}(\varphi-z), \forall \varphi \in H_{0}^{1}\left(\Omega^{\sharp}\right), \varphi \geq 0, \\
z \geq 0,
\end{array}\right.
$$

where in this case we denote by $f^{\sharp}$ the signed rearrangement of $f$, that is

$$
f^{\sharp}(x)=\left(f^{+}\right)^{\sharp}-\left(f^{-}\right)_{\sharp} \text {. }
$$

REMARK 3.1. - The solution $z$ of (30) is spherically symmetric and decreasing with respect to $\rho=|x|$. Moreover, it is the solution of the following problem

$\int_{\Omega^{\sharp}}\left[\exp (-B|x|) z_{x_{i}}(\varphi-z)_{x_{i}}+\gamma(x) z(\varphi-z)\right] \geq \int_{\Omega^{\sharp}} \exp (-B|x|) f^{\sharp}(x)(\varphi-z)$,

for all $\varphi \in H_{0}^{1}\left(\Omega^{\sharp}\right), \varphi \geq 0$.

THEOREM 3.1. - Let us assume that the coefficients of L satisfy (1), (2), (14); let $u$ and $v$ be the solutions respectively of (29) and (30). We have

$$
\int_{0}^{s} u^{*} \leq \int_{0}^{s} z^{*}, \forall s \in[0,|u>0|]
$$

and, if $\gamma(x)=0$ for $|x| \leq R_{0}$,

$$
u^{\sharp}(x) \leq z^{\sharp}(x),|x| \leq R_{0} .
$$


Proof. - As in the proof of theorem 2.1, writing $u(s)=u^{*}(s)$, we have

$$
-u^{\prime}(s) \leq k(s) \int_{0}^{s}\left(e^{-1} f^{*}-\gamma_{*} u\right), s \in[0,|u>0|[,
$$

where $f^{*}(s)=\left(f^{+}\right)^{*}-\left(f^{-}\right)_{*}$; moreover if $z(s)=z^{*}(s)$, we have

$$
\left.-z^{\prime}(s)=k(s) \int_{0}^{s}\left(e^{-1} f^{*}-\gamma_{*} z\right), s \in\right] 0,|z>0|[\text {. }
$$

We remark that if $|z>0|<|\Omega|$, then $\left(f^{-}\right)_{*} \not \equiv 0$ on $] 0,|z>0|[$ : indeed, since $z \in C^{1}(] 0,|\Omega|[)$, we have $z^{\prime}(|z>0|)=0$. Hence, we deduce from (34)

$$
\left\{\begin{array}{l}
-\left(k^{-1} z^{\prime}\right)^{\prime}+\gamma_{*} z=e^{-1} f^{*} \\
z(|z>0|)=z^{\prime}(|z>0|)=0
\end{array}\right.
$$

and the assertion follows from the maximum principle.

From (33), (34) we obtain

$$
w^{\prime}-k(s) \int_{0}^{s} \gamma_{*} w \geq 0 \text { a.e. on }[0,|u>0|],
$$

where $w=u-z$. The above inequality is obvious if $s \in \min \{|u>0|$, $|z>0|\}$. When $|z>0|<|u>0|$, we set

$$
\tilde{f}(s)= \begin{cases}f^{*}(s), & 0 \leq s \leq|z>0| \\ 0, & |z>0|<s \leq|u>0|,\end{cases}
$$

so we can replace (34) by the equation

$$
-z^{\prime}(s)=k(s) \int_{0}^{s}\left(e^{-1} \tilde{f}-\gamma_{*} z\right), s \in[0,|u>0|] .
$$

From (33), (36) we obtain

$$
w^{\prime}(s)-k(s) \int_{0}^{s} \gamma_{*} w \geq-k(s) \int_{|z>0|}^{s} e^{-1} f^{*}, s \in[|z>0|,|u>0|]
$$

since $f^{*}(s)<0$ if $s>|z>0|$ we get (35). From now on we can proceed as in the proof of theorem 2.1.

A comparison result may also be obtained when we consider the dual operator

$$
L^{*} u=-\sum_{i=1}^{N}\left(\sum_{j=1}^{N} a_{i j}(x) u_{x_{j}}\right)_{x_{i}}-\sum_{i=1}^{N}\left(b_{i} u\right)_{x_{i}}+c
$$


and the related problem for $u \in H_{0}^{1}(\Omega)$,

$$
\left\{\begin{array}{l}
\prec L^{*} u, \varphi-u \succ \geq \int_{\Omega} f(\varphi-u), \forall \varphi \in H_{0}^{1}(\Omega), \varphi \geq 0 \\
u \geq 0
\end{array}\right.
$$

where $f \in L^{2}(\Omega)$ and conditions (1), (2), (14) are fullfilled.

LEMMA 3.1. - The problem (39) has a unique solution.

Several proofs are possible. We present a direct one. Let us fix $\lambda>0$ such that

$$
\prec L^{*} \varphi, \psi \succ+\lambda(\varphi, \psi)_{L^{2}}=a^{*}(\varphi, \psi)+\lambda(\varphi, \psi)_{L^{2}}
$$

is coercive on $H_{0}^{1}(\Omega)$. By an iterative procedure we can define the following sequence: $u_{0}=0$ and $u_{n+1} \geq 0$ is the solution of

$$
\begin{aligned}
& a^{*}\left(u_{n+1}, \varphi-u_{n+1}\right)+\lambda\left(u_{n+1}, \varphi-u_{n+1}\right)_{L^{2}} \\
& \quad \geq \int_{\Omega}\left(f+\lambda u_{n}\right)\left(\varphi-u_{n+1}\right), \forall \varphi \in H_{0}^{1}(\Omega), \varphi \geq 0 .
\end{aligned}
$$

By the maximum principle $\left\{u_{n}\right\}$ is increasing. Since

$$
L^{*} u_{n+1}+\lambda u_{n+1} \leq\left(f+\lambda u_{n}\right)^{+}
$$

we have $L^{*} u_{n+1} \leq f^{+}$; then $u_{n+1} \leq U$ where $U \in H_{0}^{1}(\Omega)$ is the wcak solution of $L^{*} U=f^{+}$. Hence we deduce that $\left\{u_{n}\right\}$ converges in $L^{2}$ to $u \in H_{0}^{1}(\Omega)$; obviously $u$ is solution of (39).

Next, let $u, \widetilde{u}$ be two solutions of (39); proceeding as in [11] we obtain

$$
\|u-\widetilde{u}\|_{L^{\infty}} \leq\|V\|_{L^{\infty}}
$$

where $V \in H_{0}^{1}(\Omega)$ is solution of $L^{*} V+\lambda V=\lambda|u-\widetilde{u}|$. If $\varphi_{1}>0$ is the first eigenfunction of $L$, i.e.

$$
L \varphi_{1}=\lambda_{1} \varphi_{1}, \lambda_{1}>0, \varphi_{1}>0 \text { on } \Omega, \varphi_{1}=0 \text { on } \partial \Omega,
$$

we have

$$
\left(\lambda+\lambda_{1}\right) \int_{\Omega} V \varphi_{1}=\lambda \int_{\Omega}|u-\widetilde{u}| \varphi_{1} \leq \int_{\Omega} V \varphi_{1},
$$

from which we deduce $V=|u-\widetilde{u}|=0$. 
We now consider the following problem

$$
\left\{\begin{array}{l}
\prec S^{*} v, \varphi-v \succ \geq \int_{\Omega} f^{\sharp}(\varphi-v) \quad \forall \varphi \in I_{0}^{1}\left(\Omega^{\sharp}\right), \varphi \geq 0 \\
v \geq 0
\end{array}\right.
$$

The problem (40) has a unique solution $v \in H_{0}^{1}\left(\Omega^{\sharp}\right)$. Moreover, $v$ is spherically symmetric and decreasing with respect to $\rho=|x|$ and the inequality (28) holds for all $x$ such that $0 \leq|x| \leq\left(\frac{|v>0|}{\omega_{N}}\right)^{1 /}$

THEOREM 3.2. - Let $u$ and $v$ be the solutions of problems (39) and (40) respectively. Then we have

$$
\int_{0}^{s} u^{*} \leq \int_{0}^{s} v^{*}, \forall s \in[0,|u>0|] .
$$

Furthermore, if $\gamma(x) \equiv 0$ for $|x| \leq R_{0}$, then

$$
u^{\sharp}(x) \leq v^{\sharp}(x),|x| \leq R_{0} .
$$

First step. - In this step we provide a differential inequality satisfied by the rearrangement $u^{*}(s)=u(s)$ of the solution of (39). The procedure is largely standard (see [2], [5] for example); therefore, we only sketch the argument. For $t, h>0$ fixed, we set

$$
\theta_{h}(x):= \begin{cases}h, & u(x)>t+h \\ (u(x)-t), & t<u(x) \leq t+h \\ 0, & u(x) \leq t\end{cases}
$$

If we use $u \pm \theta_{h}$ as test function in (39), letting $h$ go to zero, we find

$$
-\frac{d}{d t} \int_{u>t} \sum_{i, j=1}^{N} a_{i j} u_{x_{i}} u_{x_{j}}=-\frac{d}{d t} \int_{u>t} u \sum_{i=1}^{N} b_{i} u_{x_{i}}+\int_{u>t}(f-c u) .
$$

Proceeding as in [2], we obtain the following inequalities, for a.e. $t \in[0, \sup u[$ :

$$
\begin{gathered}
-\frac{d}{d t} \int_{u>t} \sum_{i, j=1}^{N} a_{i j} u_{x_{i}} u_{x_{j}} \geq-\frac{d}{d t} \int_{u>t}|\nabla u|^{2} \\
-\frac{d}{d t} \int_{u>t} u \sum_{i=1}^{N} b_{i} u_{x_{i}} \leq B t\left(-\mu^{\prime}(t)\right)^{\frac{1}{2}}\left(-\frac{d}{d t} \int_{u>t}|\nabla u|^{2}\right)^{\frac{1}{2}}
\end{gathered}
$$




$$
\int_{u>t}(f-c u) \leq \int_{0}^{\mu(t)}\left(f^{*}-\gamma_{*} e u\right)
$$

where in the last inequality we used Hardy inequality and condition (14). Hence we deduce from (44)-(47)

$$
-\frac{d}{d t} \int_{u>t}|\nabla u|^{2} \leq B t\left(-\mu^{\prime}(t)\right)^{\frac{1}{2}}\left(-\frac{d}{d t} \int_{u>t}|\nabla u|^{2}\right)^{\frac{1}{2}}+F_{u}(\mu(t)),
$$

where

$$
F_{u}(s)=\int_{0}^{s}\left(f^{*}-e \gamma_{*} u\right)
$$

It is useful to remark that (44) yields

$$
\int_{u>0}(f-c u) \geq 0
$$

We next recall the following inequality that is a consequence of the classical isoperimetric inequality ( $\mathrm{scc}$ [9], [14])

$$
k_{N} \mu(t)^{1-1 / N} \leq-\frac{d}{d t} \int_{u>t}|\nabla u| \leq\left(-\mu^{\prime}(t)\right)^{\frac{1}{2}}\left(-\frac{d}{d t} \int_{u>t}|\nabla u|^{2}\right)^{\frac{1}{2}}
$$

And we obtain using (48)

$$
\left(-\frac{d}{d t} \int_{u>t}|\nabla u|^{2}\right)^{\frac{1}{2}} \leq \frac{B t}{2}\left(-\mu^{\prime}(\iota)\right)^{\frac{1}{2}}+\sqrt{\frac{B^{2} t^{2}}{4}\left(-\mu^{\prime}(t)\right)+F_{u}(\mu(t))}
$$

and then by $(50)$

$$
k_{N} \mu(t)^{1-1 / N}\left(-\mu^{\prime}(t)\right)^{-1 / 2} \leq \frac{B t}{2}\left(-\mu^{\prime}(t)\right)^{1 / 2}+\sqrt{\frac{B^{2} t^{2}}{4}-\left(-\mu^{\prime}(t)\right)+F_{u}(\mu(t))} .
$$

The above inequality can be rewritten in terms of the rearrangement $u(s)$ of the solution of (39) in the following way

$$
k_{N} s^{1-1 / N}\left(-u^{\prime}(s)\right) \leq \frac{B}{2} u(s)+\sqrt{\frac{B^{2}}{4} u^{2}+\left(-u^{\prime}(s)\right) F_{u}(s)} .
$$

Second step. - An easy computation shows that the rearrangement $v^{*}(s)=v(s)$ of the solution of problem (40) satisfies the following equation:

$$
k_{N}^{2} s^{2-2 / N}\left(-v^{\prime}(s)\right)=B k_{N} s^{1-1 / N} v(s)+F_{v}(s), s \in[0,|v>0|[.
$$


Since $F_{v}(s) \geq 0($ see $(28))$, we can write

$$
k_{N} s^{1 \cdot 1 / N}\left(-v^{\prime}(s)\right)=\frac{B}{2} v(s)+\sqrt{\frac{B^{2}}{4} v^{2}(s)+\left(-v^{\prime}(s)\right) F_{v}(s)} .
$$

REMARK 3.2. - Setting $f=f^{+}-f^{-}$, assume $f^{+}, f^{-} \not \equiv 0$. Then we have

$$
\operatorname{supp}\left(f^{+}\right)^{*} \subset[0,|v>0|] .
$$

Indeed, if we set $V(s)=e(s) v(s),(53)$ becomes

$$
-\left(k^{-1}(s) V^{\prime}(s)\right)^{\prime}=f^{*}-e(s) \gamma_{*}(s) v .
$$

Since $v^{\prime}(|v>0|)=0, V$ satisfies the following boundary conditions

$$
V(|v>0|)=V^{\prime}(|v>0|)=0
$$

Hence, by the maximum principle, we deduce that $f^{*}$ cannot be non negative on $[0,|v>0|[$.

Third step. - We set $s_{0}=\left|\left\{s \in[0,|\Omega|]: \gamma_{*}(s)=0\right\}\right|$.

We consider several cases.

i) $|u>0| \leq s_{0}$.

We thus have $|u>0| \leq|v>0|$. Indeed, let us assume that $|v>0|>|u>0|$. Since $|u>0| \leq s_{0}$, we deduce from (49)

$$
\int_{0}^{|u>0|} f^{*} \geq 0
$$

on the other side (see (53)) we have

$$
F_{v}(|v>0|)=\int_{0}^{|v>0|} f^{*}=0
$$

Since $|v>0|<|u>0|$, we obtain using Remark 3.2 .

$$
\int_{0}^{|u>0|} f^{*}<\int_{0}^{|v>0|} f^{*}=0
$$

and this leads to a contradiction.

The above arguments imply also that

$$
\left.\left.F_{u}(s)=\int_{0}^{s} f^{*}>0, \forall s \in\right] 0,|u>0|\right]
$$

Vol. 16, $n^{\circ}$ 2-1999. 
hence we can easily deduce that $u$ satisfies the following differential inequality

$$
k_{N}^{2} s^{2-2 / N}\left(-u^{\prime}(s)\right) \leq B k_{N} s^{1-1 / N} u(s)+\int_{0}^{s} f^{*} .
$$

From (55) and (53), setting $w=u-v$ we find

$$
\left.k_{N} s^{1-1 / N} w^{\prime}+B w \geq 0, s \in\right] 0,|u>0|[
$$

and then

$$
\left.(e(s) w(s))^{\prime} \geq 0, s \in\right] 0,|u>0|[
$$

integrating from $s$ to $|u>0|$, we get $v(s) \geq u(s)$.

ii) $s_{0}<|u>0| \leq|v>0|$.

\section{Letting}

$$
W(s)=\int_{s_{0}}^{s} e \gamma_{*}\left(u^{*}-v^{*}\right), s \geq s_{0}
$$

we show first that

$$
W(s) \leq 0, s \in\left[s_{0},|u>0|\right]
$$

If (56) is not true there exist $\bar{s} \in\left[s_{0},|u>0|\right]$ and $\delta>0$ such that

$$
\left\{\begin{array}{l}
W(\bar{s})>0, W^{\prime}(\bar{s}) \geq 0 \\
0<W(s)<W(\bar{s}), s \in] \bar{s}-\delta, \bar{s}[
\end{array}\right.
$$

We remark that $\bar{s} \in] s_{0},|u>0|[$. Namely if

$$
\bar{s}=|u>0|=|v>0|
$$

since $W(\bar{s})>0$, we have

$$
F_{u}(\bar{s})<F_{v}(\bar{s})=0
$$

that contradicts (49); if $\bar{s}=|u>0|<|v>0|$, we have

$$
W^{\prime}(\bar{s})=-e(\bar{s}) \gamma_{*}(\bar{s}) v(\bar{s})<0
$$

that contradicts the condition $W^{\prime}(\bar{s}) \geq 0$. 
The function $W(s)$ has right and left derivatives for all $s$. The above derivatives cannot be non positive on $[\bar{s}-\delta, \bar{s}]$. Hence there exists an interval $] \alpha, \beta[\subset] \bar{s}-\delta, \bar{s}[$ such that

$$
\left\{\begin{array}{l}
u(s)-v(s)>0, \quad s \in] \alpha, \beta[ \\
u(\beta)-v(\beta)=0
\end{array}\right.
$$

From (57) we deduce that there exists a sequence $s_{n} / \beta$ such that

$$
l_{u}=\lim _{n} u^{\prime}\left(s_{n}\right) \leq \lim _{n} v^{\prime}\left(s_{n}\right)=l_{v} \leq 0
$$

Moreover, we have $-\infty<l_{u} \leq l_{n}<0$, (see (57)).

Then we obtain using (52)

$$
k_{N} \beta^{1-1 / N} \leq \frac{B}{2} \frac{u(\beta)}{\left(-l_{u}\right)}+\sqrt{\left(\frac{B u(\beta)}{2 l_{u}}\right)^{2}-\frac{F_{u}(\beta)}{l_{u}}}
$$

while (54) implies

$$
k_{N} \beta^{1-1 / N}=\frac{B}{2} \frac{v(\beta)}{\left(-l_{v}\right)}+\sqrt{\left(\frac{B v(\beta)}{2 l_{v}}\right)^{2}-\frac{F_{v}(\beta)}{l_{v}}} .
$$

Since $u(\beta)=v(\beta), 0<-l_{v} \leq-l_{u}$ and

$$
F_{u}(\beta)=\int_{0}^{\beta}\left(f^{*}-e \gamma_{*} u\right)<\int_{0}^{\beta}\left(f_{*}-e \gamma_{*} v\right)=F_{v}(\beta)
$$

(58) and (59) yield a contradiction.

Hence,

$$
W(s) \leq 0, s \in\left[s_{0},|u>0|\right]
$$

as in the previous case we then deduce that

$$
\int_{s_{0}}^{s} u^{*} \leq \int_{s_{0}}^{s} v^{*}, s \in\left[s_{0},|u>0|[.\right.
$$

iii) $|v>0|<|u>0|$.

The function $W(s)$ is increasing on $\left[\max \left\{|v>0|, s_{0}\right\},|u>0|\right]$, hence either $s_{0} \leq|v>0|$ or $s_{0}=|u>0|$. The case $s_{0}=|v>0|$ can be analyzed as the previous one. Let $s_{0}$ be $|u>0|$; from (54) we deduce that 
$F_{v}(|v>0|)=0$. Since $f^{*}(s)<0$ when $s \in[|v>0|,|u>0|]$, ( see Remark 2.1 ), we get $F_{v}(s)<0$ for $s>|v>0|$. As $W(|u>0|)>0$ it follows

$$
0>F_{v}(|u>0|)>F_{u}(|u>0|) \text {. }
$$

a contradiction since $F_{u}(|u>0|) \geq 0$.

Arguing as in the previous case we obtain (41). Moreover we deduce from (52), (53) that we have for $s \in\left[0, s_{0}[\right.$,

$$
\begin{aligned}
& k_{N}^{2} s^{2-2 / N}\left(-u^{\prime}(s)\right) \leq B k_{N} s^{1-1 / N} u(s)+\int_{0}^{s} f^{*}, \\
& k_{N}^{2} s^{2-2 / N}\left(-v^{\prime}(s)\right)=B k_{N} s^{1-1 / N} v(s)+\int_{0}^{s} f^{*} .
\end{aligned}
$$

Since $u\left(s_{0}\right) \leq v\left(s_{0}\right)$, proceeding as in the case i) we obtain the estimate (42).

REMARK 3.3. - As a consequence of theorem (3.2) we have

$$
F_{v}(s) \leq F_{u}(s), s \in[0,|u>0|]
$$

Since $F_{v} \geq 0$ we have $F_{u}(s) \geq 0$ so $u^{*}(s)=u(s)$ verifies the following differential inequality

$$
k_{N}^{2} s^{2-2 / N}\left(-u^{\prime}(s)\right) \leq B k_{N} s^{1-1 / N} u(s)+F_{u}(s) .
$$

\section{APPENDIX}

We prove here the differential inequality stated in the previous sections. For the sake of simplicity we consider the case of an elliptic equation and we assume $f$ (and then $u$ ) to be non negative.

REMARK 4.1. - Let $\mu$ denote the distribution function of a non negative function $u \in H_{0}^{1}(\Omega)$; we have

- $\mu:[0, \sup u] \rightarrow[0,|\Omega|]$ is strictly decreasing;

- $\left.u^{*}(\mu(t))=t, \forall t \in\right] 0, \sup u[$;

- $u^{*}$ is absolutely continuous on every compact subset of $] 0,|\Omega|[$.

LEMMA 4.1. - Let $u(s)=u^{*}(s)$ denote the decreasing rearrangement of a function $u \in H_{0}^{1}(\Omega)$; if

$$
I_{0}=\{s \in] 0,|\Omega|\left[: \frac{d u}{d s}=0\right\}
$$


and $J \subset] 0, \sup u[$ has measure zero, we have

$$
\left|u^{-1}(J)-I_{0}\right|=0
$$

Indeed, if $J=\cap_{n} I_{n}$ where $\left\{I_{n}\right\}$ is a decreasing sequence of open sets, we have

$$
\lim _{n}\left|I_{n}\right|=\lim _{n} \int_{u^{-1}\left(I_{n}\right)} u u^{\prime}=\lim _{n} \int_{u^{-1}\left(I_{n}\right)-I_{0}} u^{\prime}=\int_{u^{-1}(J)-I_{0}} u^{\prime} .
$$

As $\lim _{n}\left|I_{n}\right|=0$ it is

$$
\int_{u^{-1}(J)-I_{0}} u^{\prime}=0
$$

so we get (60) because $u^{\prime}(s)>0$ when $s \in u^{-1}(J)-I_{0}$. The general case can be obtained because every set $J$ such that $|J|=0$ is a subset of $G_{\delta}$ set whose measure is zero.

$$
\begin{gathered}
\text { LEMMA 4.2. - If } F(s)=\int_{0}^{s} f \text { with } f \in L^{1} \text {, we have for a.e. } t \\
\frac{d F(\mu(t))}{d t}=f(\mu(t)) \mu^{\prime}(t)
\end{gathered}
$$

Obviously $\mu$ is a.e. differentiable; moreover

$$
F^{\prime}(s)=f(s) \quad \forall s \in[0,|\Omega|]-S_{0}
$$

with $\left|S_{0}\right|=0$. The set of values $t$ where $\mu$ is differentiable and $\mu(t) \in S_{0}$ has measure zero in view of Lemma 4.1. And we conclude.

We can now prove the following differential inequality, satisfied by the solution $u \in H_{0}^{1}(\Omega)$ of problem (10):

$$
\left.-u^{\prime}(s) \leq k(s) \int_{0}^{s} e^{-1}\left(f^{*}-c_{*} u\right), \text { a.e. on }\right] 0,|\Omega|[\text {. }
$$

The first part of the proof uses standard arguments (see [2]). By using appropriate test functions we obtain, a.e. on $] 0, \sup u[$, the following inequality :

$$
-\frac{d}{d t} \int_{u>t}|\nabla u|^{2} \leq \int_{u>t}\left(f-c u-\sum b_{i} u_{x_{i}}\right) .
$$

Vol. 16, n 2-1999. 
Using the isoperimetric inequalities and Hardy inequality we deduce

$$
\begin{gathered}
-\frac{d}{d t} \int_{u>t}|\nabla u|^{2} \\
\leq \frac{B}{k_{N}} \int_{t}^{+\infty} \mu^{-1+1 / N}\left(-\mu^{\prime}\right)\left(-\frac{\mathrm{d}}{\mathrm{d} \tau} \int_{u>\tau}|\nabla u|^{2}\right)+\int_{0}^{\mu(t)}\left(f^{*}-c_{*} u\right),
\end{gathered}
$$

for a.e. $t$. We denote by

$$
\psi(t)=-\frac{d}{d t} \int_{u>t}|\nabla u|^{2}, F(s)=\int_{0}^{s}\left(f^{*}-c_{*} u\right)
$$

and we write

$$
\psi(t) \leq \frac{B}{k_{N}} \int_{t}^{+\infty} \mu^{-1+1 / N}\left(-\mu^{\prime}\right) \psi+F(\mu(t)),
$$

where $t \in] 0, \sup u[-J$, and $|J|=0$. We extend $\psi$ on $] 0, \sup u[$ setting $\psi=0$ on $J$ : we denote by $\varphi$ this extension.

REMARK 4.2. - The function $\varphi((u(s))$ is bounded. Indeed, we can assume that $u\left(I_{0}\right) \subset J$, because $\left|u\left(I_{0}\right)\right|=0$; so $\varphi(u(r))=0$ if $r \in I_{0}$. When $r \notin I_{0}$, by Lemma (4.1), we have for a.e. $] 0,|\Omega|\left[-I_{0}, \varphi(u(r))=\psi(u(r))\right.$; hence from (62) we get

$$
|\varphi(u(r))| \leq B\|\nabla u\|_{L^{1}}+\|f\|_{L^{1}} .
$$

REMARK 4.3. - If $g \in L^{1}$ a non negative function; then

$$
\int_{t}^{+\infty} g(\mu(\tau))\left(-\mu^{\prime}(\tau)\right) d \tau \leq \int_{0}^{\mu(t)} g
$$

Indeed, we deduce from Lemma (4.2)

$$
g(\mu(t))\left(-\mu^{\prime}(t)\right)=\frac{d}{d t} \int_{0}^{\mu(t)} g .
$$

We write (63) in terms of $\varphi$

$$
\varphi(t) \leq \frac{B}{k_{N}} \int_{t}^{+\infty} \varphi(u(\mu(\tau))) \mu^{-1+1 / N}(\tau)\left(-\mu^{\prime}(\tau)\right) \mathrm{d} \tau+F(\mu(t))
$$

hence by Remark 4.3 we get

$$
\varphi(t) \leq \frac{B}{k_{N}} \int_{0}^{\mu(t)} \varphi(u(r)) r^{-1+1 / N} \mathrm{~d} r+F(\mu(t)) .
$$


Setting

$$
H(s)=\frac{B}{k_{N}} \int_{0}^{s} \varphi(u(r)) r^{-1+1 / N} \mathrm{~d} r
$$

we have

$$
\varphi(u(s)) \leq H(s)+F(s)=G(s)
$$

The above inequality holds a.c. $] 0,|\Omega|\left[-I_{0}\right.$ since (66) bccomes (65) for $s=\mu(t)$.

If $s \in I_{0}$ either $s$ is a point of accumulation of the set $] 0,|\Omega|\left[-I_{0}\right.$ or $s$ belongs to the interior of $I_{0}$. In the first case there exists a sequence $\left\{s_{n}\right\}$ with $s_{n}$ such that $G\left(s_{n}\right) \geq \varphi\left(u\left(s_{n}\right)\right) \geq 0$. Since $G$ is continuous, we deduce

$$
G(s)=\lim _{n} G\left(s_{n}\right) \geq 0=\varphi(u(s)) .
$$

In the second case, we can easily prove that $s \in] s_{1}, s_{2}\left[\subset I_{0}\right.$ and $s_{1}, s_{2}$ satisfies (66). Since $u$ is constant on $] s_{1}, s_{2}$ [, we see that $\varphi(u(s))$ and $H(s)$ are constant on $] s_{1}, s_{2}[$. Moreover

$$
\left.F(s)=\int_{0}^{s} f^{*}-u\left(s_{1}\right) \int_{s_{1}}^{s} c_{*}-\int_{0}^{s_{1}} c_{*} u, s \in\right] s_{1}, s_{2}[
$$

hence $F$ is concave on $] s_{1}, s_{2}\left[\right.$. Since $G\left(s_{1}\right), G\left(s_{2}\right) \geq 0$, we have $G(s) \geq 0$ on $] s_{1}, s_{2}$ [ and then (66) is proved.

From (66), by Gronwall inequality, we get

$$
0 \leq \varphi(u(s)) \leq e(s) \int_{0}^{s} e^{-1}\left(f^{*}-c_{*} u\right) .
$$

On the other side we have a.e. on $] 0,|\Omega|\left[-I_{0}\right.$

$$
\varphi(u(s))=\psi(u(s))=-\left.\frac{\mathrm{d}}{\mathrm{d} t} \int_{u>t}|\nabla u|^{2}\right|_{t=u(s)} \geq-u^{\prime}(s) k_{N}^{2} s^{2-2 / N} .
$$

Therefore we have

$$
-u^{\prime}(s) \leq k(s) \int_{0}^{s} e^{-1}\left(f^{*}-c_{*} u\right)
$$

Finally, the previous inequality holds on $I_{0}$, since the left hand side is non negative. And (61) is proved. 


\section{REFERENCES}

[1] A. Alvino, P.-L. Lions and G. Trombetti, On optimization problems with prescribed rearrangements, Nonlin. An. TMA, Vol. 13, 1989, pp. 185-220.

[2] A. Alvino, P.-L. Lions and G. Trombetti, Comparison results for elliptic and parabolic equations via Schwarz symmetrization, Ann. I.H.P. An. Non Lin., Vol. 7, 1990, pp. 37-65.

[3] A. Alvino, P.-L. Lions and G. Trombetti, Comparison results for elliptic and parabolic equations via symmetrization: a new approach, Diff. and Int. Eq., Vol. 4, 1991, pp. 25-50.

[4] A. Alvino and G. Trombetri, Equazioni ellittiche con termini di ordine inferiore e riordinamenti, Atti Acc. Naz. Lincei Rend. Fis., s.8, Vol. 66, 1979, pp. 194-200.

[5] A. Alvino, S. Matarasso and G. Trombetti, Variational inequalities and rearrangements, Rend. Mat. Acc. Lincei, s.9, Vol. 3, 1992, pp. 271-285.

[6] C. BANDLE, Isoperimetric inequalities and applications, Monographs and Studies in Math., Pitman, London, 1980.

[7] C. BAndLE and J. Mossino, Rearrangements in variational inequalities, Ann. Mat. Pura e Appl., Vol. 138, 1984, pp. 1-14.

[8] G. ChIm, Norme di Orlicz delle soluzioni di una classe di equazioni ellittiche, Boll. U.M.I., Vol. 16-A, 1978, pp. 179-185

[9] E. DE GIoRGi, Su una teoria generale della misura $r$-1-dimensionale in uno spazio ad $r$ dimensioni, Ann. Mat. Pura e Appl,, Vol. 36, 1954, pp. 191-213.

[10] G. H. Hardy, J. E. Littlewood and G. Polya, Inequalities, Cambridge, Univ. Press, 1964.

[11] P.-L. Lions, A remark on some elliptic second order problems, Boll. U.M.I., (5) Vol. 17-A, 1980, pp. 267-270.

[12] P.-L. Lions, Non linear partial differential equations and their appl., College de France, Semin., 1, Pitman, London, 1980, pp. 308-319.

[13] C. MAdERnA and S. SALSA, Some special properties of solutions to obstacle problem, Rend. Sem. Mat. Un. Padova, Vol. 71, 1984, pp. 121-129.

[14] G. TALENTI, Elliptic equations and rearrangements, Ann. Sc. Norm. Sup. Pisa, Vol. 3, 1976, pp. 697-718.

[15] G. TALENTI, Linear elliptic P.D.E.'s: level sets, rearrangements and a priori estimates of solutions, Boll. UMI, 4-B, 1985, pp. 917-949.

(Manuscript received February 3, 1997.) 